\title{
Energy level structure of the ground configuration in the $\mathrm{Er}^{3+}$ free ion
}

\author{
L. Radžiūtè ${ }^{1}$, D. Kato ${ }^{2,3}$, G. Gaigalas ${ }^{1,2}$, P. Jönsson ${ }^{4}$, P. \\ Rynkun $^{1}$, V. Jonauskas ${ }^{1}$ and S. Kučas ${ }^{1}$ \\ ${ }^{1}$ Institute of Theoretical Physics and Astronomy, Vilnius University \\ A. Goštauto 12, LT-01108 Vilnius, Lithuania \\ 2 National Institute for Fusion Science, 322-6 Oroshi-cho, Toki 509-5292, Japan \\ 3 Department of Fusion Science, The Graduate University of Advanced Studies \\ (SOKENDAI), Toki, Gifu 509-5292, Japan \\ 4 Materials Science and Applied Mathematics, Malmö University, 20506 Malmö, \\ Sweden \\ E-mail: Laima.Radziute@tfai.vu.lt
}

\begin{abstract}
Energy levels of the ground configuration $[\mathrm{Xe}] 4 f^{11}$ in the $\mathrm{Er}^{3+}$ ion are reported from relativistic configuration interaction calculations. Calculations are performed using the relativistic atomic structure package GRASP2K, which implements the multiconfiguration Dirac-Hartree-Fock method. The Breit transverse interaction and leading QED effects are included as perturbations. The final energies of 41 levels are compared with results from experiment and semi-empirical methods.
\end{abstract}

PACS numbers: 31.15.A-, 31.15.ve, 31.15.xr

Submitted to: Physica Scripta

Keywords: energy structure, erbium, relativistic configuration interaction, multiconfiguration Dirac-Hartree-Fock 


\section{Introduction}

$\mathrm{Er}_{2} \mathrm{O}_{3}$ is a good candidate for blanket system in fusion reactors, because of its chemical and physical properties [1]. However, even for free ions of $\mathbf{E r}^{3+}$, spectroscopic data are available only for 12 lowest levels of ground state configuration. Energy levels of free $\mathrm{Er}^{3+}$ were obtained by Carter from emission spectra of a highcurrent spark [2], however, most authors [3, 4, 5, 6] used semi-empirical methods. The idea of this work is to calculate all 41 levels of the $[\mathrm{Xe}] 4 f^{11}$ ground configuration in $\mathrm{Er}^{3+}$ using an ab initio approach. In our previous work [7] the 12 lowest levels of the ground configuration were calculated. The good accuracy of these data (the discrepancy with experiment is less than 6.9\%) encouraged us to compute higher levels using the previously described method for accounting for electron correlations effects. Our results were obtained with the multiconfiguration Dirac-HartreeFock (MCDHF) and relativistic configuration interaction (RCI) [8] methods, using the GRASP2K (A General-Purpose Relativistic Atomic Structure Program) code [9].

\section{Computational procedure}

7 The MCDHF method for computing energy levels has been reviewed by Grant [10], and 18 here we just give a brief outline.

\subsection{Multiconfiguration Dirac-Hartree-Fock}

In this method wave functions of fine-structure states are approximated by atomic state functions. The atomic state functions (ASFs) are expanded in symmetry adapted configuration state functions (CSFs)

$$
\Psi\left(K \gamma J M_{J}\right)=\sum_{K^{\prime} \gamma^{\prime}}^{N_{C S F s}} c_{K^{\prime} \gamma^{\prime}} \Phi\left(K^{\prime} \gamma^{\prime} J M_{J}\right)
$$

In the expression above, $J$ and $M_{J}$ are the angular quantum numbers and $\gamma^{\prime}$ denotes a state of configuration $K^{\prime}$. The $K$ and $\gamma$ are labels of the reference configuration and the reference state. The CSFs are anti-symmetrized and coupled products of one-electron Dirac orbitals. In the relativistic self-consistent field (RSCF) procedure both the radial parts of the Dirac orbitals and the expansion coefficients of the CSFs are optimized to self-consistency with respect to an energy functional built on the Dirac-Coulomb Hamiltonian

$$
H_{\mathrm{DC}}=\sum_{i=1}^{N}\left(c \alpha_{i} \cdot \mathbf{p}_{i}+\left(\beta_{i}-1\right) c^{2}+V_{i}^{N}\right)+\sum_{i>j}^{N} \frac{1}{r_{i j}}
$$

where $V^{N}$ is the monopole part of the electron-nucleus Coulomb interaction. The transverse Breit interaction 


$$
\begin{aligned}
& H_{\text {Breit }} \\
& =-\sum_{i<j}^{N}\left[\alpha_{i} \cdot \alpha_{j} \frac{\cos \left(\omega_{i j} r_{i j} / c\right)}{r_{i j}}\right. \\
& \left.\quad+\left(\alpha_{i} \cdot \nabla_{i}\right)\left(\alpha_{j} \cdot \nabla_{j}\right) \frac{\cos \left(\omega_{i j} r_{i j} / c\right)-1}{\omega_{i j}^{2} r_{i j} / c^{2}}\right]
\end{aligned}
$$

as well as leading QED corrections, vacuum polarization and self-energy, can be included in subsequent RCI calculations [11], where now only the expansion coefficients are optimized. Calculations can be done for single states, but also for portions of a spectrum in the extended optimal level (EOL) scheme, where optimization is on a weighted sum of energies. Using the latter scheme a balanced description of a number of fine-structure states belonging to one or more configurations can be obtained in a single calculation. All calculations were performed with GRASP2K [9] in which for calculations of spinangular parts of matrix elements the second quantization method in coupled tensorial form and the quasispin technique [12] were adopted.

In relativistic calculations the ASFs are given in $j j$-coupling. To adhere to the labeling conventions used by the experimentalists, the ASFs are transformed from $j j$ coupling to $L S$-coupling using the methods developed in [13, 14].

\subsection{Configuration interaction strength}

The selection of the configurations and the corresponding CSFs that enter the calculations is a crucial step in multiconfiguration methods. The method of analyzing configuration interaction strength (CIS) $[15,16]$ has been employed to find the most important admixed configurations for the considered ground configuration of $\mathrm{Er}^{3+}$. The same approach has been successfully applied for the investigation of Auger cascades $[17,18,19]$, electric dipole [20] and magnetic dipole [21, 22] transitions.

The CIS:

$$
T\left(K, K^{\prime}\right)=\frac{\sum_{\gamma \gamma^{\prime}}\left\langle\Phi(K \gamma)|H| \Phi\left(K^{\prime} \gamma^{\prime}\right)\right\rangle^{2}}{\bar{E}\left(K, K^{\prime}\right)^{2}},
$$

divided by the statistical weight $g(K)$ of the studied configuration $K\left([\mathrm{Xe}] 4 f^{11}\right)$ has the meaning of the average weight of the admixed configuration $K^{\prime}$ in the expansion of the wave functions for $K$. The larger the $T\left(K, K^{\prime}\right) / g(K)$ value, the larger the influence of the admixed configuration $K^{\prime}$ to the energy levels of the considered [Xe] $4 f^{11}$ configuration. The summation in (4) is performed over all states $\gamma$ and $\gamma^{\prime}$ of the configurations $K$ and $K^{\prime}$, respectively. The list of the admixed configurations is built by taking into account single and double excitations from the $[\mathrm{Xe}] 4 f^{11}$ configuration. A singleconfiguration pseudorelativistic method [23] is then applied to obtain radial orbitals for the corresponding configurations and $\left\langle\Phi(K \gamma)|H| \Phi\left(K^{\prime} \gamma^{\prime}\right)\right\rangle$ is the interconfiguration 
matrix element of the pseudorelativistic Hartree-Fock Hamiltonian $H$ [23]. $\bar{E}\left(K, K^{\prime}\right)$ is the average energy distance between the configurations:

$$
\begin{aligned}
& \bar{E}\left(K, K^{\prime}\right) \\
& =\frac{1}{\sum_{\gamma \gamma^{\prime}}\left\langle\Phi(K \gamma)|H| \Phi\left(K^{\prime} \gamma^{\prime}\right)\right\rangle^{2}} \\
& \quad \times \sum_{\gamma \gamma^{\prime}}\left[\langle\Phi(K \gamma)|H| \Phi(K \gamma)\rangle-\left\langle\Phi\left(K^{\prime} \gamma^{\prime}\right)|H| \Phi\left(K^{\prime} \gamma^{\prime}\right)\right\rangle\right] \\
& \quad \times\left\langle\Phi(K \gamma)|H| \Phi\left(K^{\prime} \gamma^{\prime}\right)\right\rangle^{2} .
\end{aligned}
$$

\section{Calculations}

In this work calculations were done by configuration, i.e. wave functions for all states belonging to the ground configuration were determined simultaneously in an EOL calculation. The energy functional, on which the orbitals were optimized, was the weighted energy average of the two lowest states of $[\mathrm{Xe}] 4 f^{11}$ with $J=1 / 2$, the six lowest states with $J=3 / 2$, the seven lowest states with, respectively, $J=5 / 2,7 / 2,9 / 2$, the five lowest states with $J=11 / 2$, the three lowest states with $J=13 / 2$, three lowest with $J=15 / 2$, and finally the lowest state with $J=17 / 2$.

Two sets of calculations were performed using different strategies for selecting the CSFs. In the first strategy, called the SD $\mathbf{C}+\mathbf{V}+\mathbf{C V}$ strategy, the CSFs were generated by single (S) excitations from the core (C) shells with $n=3 \ldots 5$ and from the valence (V) shell $4 f$ of the reference configuration to orbitals in active sets up to principal quantum numbers $n=6$ and angular symmetries $s . . . h$. Double (D) excitations were restricted in such a way, that one excitation would be from the core and another from the valence shell (CV) more details can be found in [7, 27]. The radial orbitals were calculated using smaller active sets generated with only S excitation. In the second strategy, based on the analysis of CIS, 3461 configurations were generated by including all SD excitations from the core shells with $n=3 \ldots 5$ and from the valence shell $4 f$ of the ground configuration to virtual orbitals with principal quantum numbers up to $n=7$ and with angular symmetries $l=s \ldots g$. The configuration list included all type of correlations: valence, core, valence-valence, core-core, core-valence. In the second step a ranking of the configurations was done based on the CIS parameter. Guided by previous work [7] only configuration with $T\left(K, K^{\prime}\right) / g(K)$ larger than $4.293 \times 10^{-6}$ were retained, leading to 183 configurations. These configurations were then used for the final calculations.

\section{Results and evaluation of data}

Table 1 compares computed energy levels with results from other theories and experiment. Levels are notated in the form ${ }^{(2 S+1)} L_{J}^{N r}$ where, instead of the group labels 
Table 1. Comparison of calculated $\left(\mathrm{Th}^{a}{ }^{a}\right.$ and $\left.\mathrm{Th}^{b}{ }^{b}\right)$ energy levels with values from semi-empirical methods and experiment (Exp). Contributions of the Dirac-Coulomb (DC), BREIT, and QED interactions to the energy (in $\mathrm{cm}^{-1}$ ) are presented. All energies are relative to the ground state.

\begin{tabular}{|c|c|c|c|c|c|c|c|c|c|c|c|}
\hline \multirow[t]{2}{*}{$L S J$} & \multirow{2}{*}{$\frac{\text { Th. }^{a}}{\text { Total }}$} & \multicolumn{4}{|c|}{$\mathrm{Th}^{b}$} & \multicolumn{4}{|c|}{ Semi-empirical } & \multirow{2}{*}{\multicolumn{2}{|c|}{$\begin{array}{cr}\text { Exp. } & \text { NIST } \\
{[2]} & {[28]}\end{array}$}} \\
\hline & & $\overline{\mathrm{DC}}$ & 3REIT Q & ED & Total & {$[3]$} & [4] & {$[6]$} & {$[5]$} & & \\
\hline${ }^{4} I_{15 / 2}^{1}{ }^{*}$ & 0 & 0 & 0 & 0 & 0 & 0 & 0 & 0 & 0 & 0 & 0 \\
\hline${ }^{4} I_{13 / 2}^{1}{ }^{*}$ & 6311 & 6646 & -281 & 4 & 6369 & 6540 & 6502 & 6405 & 6511 & 6485 & 6480 \\
\hline${ }^{4} I_{11 / 2}^{1} *$ & 10165 & 10317 & -254 & 5 & 10067 & 10123 & 10125 & 10022 & 10043 & 10123 & 10110 \\
\hline${ }^{4} I_{9 / 2}^{1} *$ & 12841 & 12612 & -83 & 4 & 12534 & 12328 & 12340 & 12241 & 12003 & 12345 & 12350 \\
\hline${ }^{4} F_{9 / 2}^{1}{ }^{*}$ & 16425 & 16137 & 70 & 1 & 16209 & 15266 & 15181 & 15076 & 14913 & 15182 & 15180 \\
\hline${ }^{4} S_{3 / 2}^{1} *$ & 20089 & 19121 & 301 & 0 & 19423 & 18433 & 18427 & 18320 & 18018 & 18299 & 18290 \\
\hline${ }^{2} H_{11 / 2}^{2}$ * & 20748 & 20140 & -68 & 2 & 20075 & 19166 & 19284 & 19175 & 18851 & 19010 & \\
\hline${ }^{4} F_{7 / 2}^{1}{ }^{1 / 2}$ & 21835 & 21841 & -41 & 3 & 21803 & 20524 & 20327 & 20123 & 20034 & 20494 & 20400 \\
\hline${ }^{4} F_{5 / 2}^{1} *$ & 23647 & 23579 & 9 & 3 & 23591 & 22065 & 21990 & 21870 & 21713 & 22181 & 22070 \\
\hline${ }^{4} F_{3 / 2}^{1} *$ & 24074 & 23692 & 85 & 3 & 23780 & 22477 & 22344 & 22227 & 21978 & 22453 & 22410 \\
\hline${ }^{2} G_{9 / 2}^{1 / 2} *$ & 25987 & 25490 & -321 & 6 & 25174 & 24539 & 24537 & 24322 & 23874 & 24475 & \\
\hline${ }^{4} G_{11 / 2}^{1}$ * & 28291 & 28487 & -238 & 3 & 28252 & 26615 & 26447 & 26327 & 25929 & 26376 & \\
\hline${ }^{4} G_{9 / 2}^{1}$ & 29369 & 29527 & -37 & 3 & 29493 & 27663 & 27431 & 27305 & & & \\
\hline${ }^{2} K_{15 / 2}^{1}$ & 29713 & 29657 & -160 & 4 & 29501 & 27041 & 27293 & 27176 & & & \\
\hline${ }^{2} G_{7 / 2}^{1}$ & 30255 & 30404 & -321 & 2 & 30085 & & 27994 & 27877 & & & \\
\hline${ }^{2} P_{3 / 2}^{1}$ & 33797 & 32952 & -121 & 5 & 32836 & & 31605 & 31477 & & & \\
\hline${ }^{2} K_{13 / 2}^{1}$ & 34993 & 34557 & 51 & 3 & 34611 & & 32521 & 32392 & & & \\
\hline${ }^{4} G_{5 / 2}^{1}$ & 35584 & 35878 & -517 & 5 & 35366 & & 33315 & 33178 & & & \\
\hline${ }^{2} P_{1 / 2}^{1}$ & 35829 & 36018 & -229 & 7 & 35796 & & & 33336 & & & \\
\hline${ }^{4} G_{7 / 2}^{1}$ & 35959 & 36218 & -374 & 5 & 35849 & 28110 & 33918 & 33783 & & & \\
\hline${ }^{2} D_{5 / 2}^{1}$ & 37550 & 36920 & -150 & 5 & 36775 & & 34794 & 34641 & & & \\
\hline${ }^{2} H_{9 / 2}^{2}$ & 37974 & 37970 & -875 & 10 & 37105 & & 36408 & 36268 & & & \\
\hline${ }^{4} D_{5 / 2}^{1}$ & 42247 & 41741 & 256 & 0 & 41997 & & 38649 & 38526 & & & \\
\hline${ }^{4} D_{7 / 2}^{1}$ & 43125 & 43375 & 175 & 1 & 43551 & & 39205 & 39067 & & & \\
\hline${ }^{2} I_{11 / 2}^{1}$ & 44363 & 44323 & -532 & 9 & 43799 & & 40309 & 40164 & & & \\
\hline${ }^{2} L_{17 / 2}^{1}$ & 44686 & 45413 & -107 & 1 & 45307 & & 40664 & 40508 & & & \\
\hline${ }^{2} D_{3 / 2}^{1}$ & 44985 & 46618 & -1 & 3 & 46619 & & 42199 & 42802 & & & \\
\hline${ }^{4} D_{3 / 2}^{1}$ & 46361 & 47489 & -531 & 2 & 46961 & & 42946 & 42044 & & & \\
\hline${ }^{2} I_{13 / 2}^{1}$ & 46509 & 47931 & -531 & 4 & 47404 & & 42947 & 42797 & & & \\
\hline${ }^{4} D_{1 / 2}^{1}$ & 50552 & 51541 & -343 & 6 & 51204 & & & 46808 & & & \\
\hline${ }^{2} L_{15 / 2}^{1}$ & 50695 & 52999 & -222 & 3 & 52780 & & 46836 & 46667 & & & \\
\hline${ }^{2} H_{9 / 2}^{1}$ & 51680 & 53002 & -199 & 5 & 52807 & & & 46989 & & & \\
\hline${ }^{2} D_{5 / 2}^{2 / 2}$ & 52987 & 53685 & -798 & 6 & 52892 & & & 48873 & & & \\
\hline${ }^{2} H_{11 / 2}^{1}$ & 54438 & 56141 & -626 & 6 & 55521 & & & 50061 & & & \\
\hline${ }^{2} D_{3 / 2}^{2}$ & 58851 & 58335 & -86 & 3 & 58252 & & & 54910 & & & \\
\hline${ }^{2} F_{7 / 2}^{2}$ & 60100 & 59478 & -633 & 9 & 58854 & & & 55055 & & & \\
\hline${ }^{2} F_{5 / 2}^{2}$ & 68234 & 67812 & -602 & 9 & 67218 & & & 62909 & & & \\
\hline${ }^{2} G_{7 / 2}^{2}$ & 72071 & 73630 & 48 & 0 & 73679 & & & 64688 & & & \\
\hline${ }^{2} G_{9 / 2}^{2}$ & 75942 & 77955 & -392 & 3 & 77566 & & & 68765 & & & \\
\hline${ }^{2} F_{5 / 2}^{1}$ & 103064 & 101651 & 139 & & 101789 & & & 93134 & & & \\
\hline${ }^{2} F_{7 / 2}^{1}$ & 107321 & 107053 & -237 & 3 & 106819 & & & 96726 & & & \\
\hline
\end{tabular}

${ }^{a} \mathrm{SD} \mathrm{C}+\mathrm{V}+\mathrm{CV}$ strategy

${ }^{b}$ CIS strategy 
$\nu W U$, single character "numbers" $N r$ are used [26]. Previous work [7] showed that $L S$ coupling is more preferable for labeling than $j j$-coupling for the ground configuration. Labels for ASFs usually are assigned as the label of the CSF making the largest contribution to the composition but such labels may not be unique. An algorithm that has been proposed for assigning unique labels [29] starts with a set of, say $m$ ASFs of the same $J$ and the $m$ CSFs with large expansion coefficients. Of the CFSs in the set the one with the largest expansion coefficient of all $m$ ASFs defines label of the ASF in which it occurs. The labeled ASF and the associated CSF are eliminated from further consideration. Each assignment gives the CSFs with the largest expansion coefficient to an ASF as the label. In this scheme, the last remaining label may be based on a contribution that is not the largest.

The levels we identify with ${ }^{2} G_{9 / 2}^{1}$ (composition: $18 \%+23 \%{ }^{2} H^{2}+21 \%{ }^{4} F^{1}+$ $\left.15 \%{ }^{2} G^{2}+11 \%{ }^{4} I^{1}\right)$ and ${ }^{4} G_{9 / 2}^{1}\left(80+12 \%{ }^{2} H^{2}\right)$ were originally identified in $[3,4,5]$ with ${ }^{2} H_{9 / 2}$ and ${ }^{2} G_{9 / 2}$ respectively. In a similar way the level we identify as ${ }^{2} H_{9 / 2}^{2}$ (composition: $\left.31 \%+24 \%{ }^{2} G^{1}+16 \%{ }^{2} G^{2}+14 \%{ }^{4} G^{1}\right)$ was originally identified with ${ }^{4} G_{9 / 2}$ in paper [4]. Our identifications agree with semi-empirical results of Weber [6].

The SD C $+\mathrm{V}+\mathrm{CV}$ strategy calculations (see column 'Th. ${ }^{a}$ ') give the positions of the first three levels in agreement with experiment. For the higher levels the agreement is less satisfactory. For calculations performed using the CIS strategy (see column 'Th. ${ }^{\text {' }}$ ) contributions to the excitation energy arising from different parts of the Hamiltonian are presented separately. Theoretical energy values for 12 lowest levels (from to ${ }^{4} I_{15 / 2}^{1}$ to $\left.{ }^{4} G_{11 / 2}^{1}\right)$ marked by "*" are given by [7]. Using the CIS strategy improves the agreement of energy levels with up to 2.6\%. As expected, the DiracCoulomb contribution is the largest, with the transverse photon (Breit) interaction giving a signicant correction. The vacuum polarization and self-energy corrections (QED) are less important.

Experimental data for centers of gravity of Stark manifolds were obtained by measuring Stark levels of the $\mathrm{Er}^{3+}$ absorption spectrum in $\mathrm{LaF}_{3}$ [3]. In the paper [4] the authors have extended the measured absorption spectrum in the same crystal into the ultraviolet region up to $2000 \AA$. With the help of small variations of parameters $\left(F_{2}, F_{4}, F_{6}\right.$ and $\left.\zeta\right)$, originally described by Wybourne [30], the free ion spectrum $[3,4]$ was determined from the experimental centers of gravity data. The eigenstates and the corresponding energy levels [6] were found (in LSJ coupling) by diagonalizing the interaction matrix defined by the spin-orbit and electrostatic energies. The spinorbit parameter $\zeta$ and the Racah parameters $E^{1}, E^{2}, E^{3}$ were determined in a fitting procedure in which centers of gravity were taken from [3] and [4]. Semi-empirical data for the spectrum in [5] were evaluated by measuring the $\mathrm{Er}^{3+}$ center of gravity in $\mathrm{ZnGa}_{2} \mathrm{O}_{4}$. Experimental data of free ion $\mathrm{Er}^{3+}$ were obtained by Carter [2] from emission spectra of a high-current spark. Energy levels recommended by NIST (National Institute of Standards and Technology) [28] were derived from the spectrum of $\mathrm{Er}^{3+}$ in $\mathrm{LaF}_{3}$ crystal. In the last column of the tables the energies from NIST [28] are given. Comparing with NIST our energy levels agrees to within $9.8 \%$ for the SD $\mathbf{C}+\mathbf{V}+\mathbf{C V}$ strategy and 
better than $6.9 \%$ for the CIS strategy. It should be noted that order of levels agree with the one given in the NIST database.

\section{Conclusions}

The MCDHF and RCI methods were used to compute the energy spectrum of the $[\mathrm{Xe}] 4 f^{11}$ configuration in $\mathrm{Er}^{3+}$. Comparing with NIST recommended values we see that our ab initio calculations for the energy spectrum agrees to within $9.8 \%$ for the free ion in the $\mathbf{S D} \mathbf{C}+\mathbf{V}+\mathbf{C V}$ strategy and better than $6.9 \%$ in the CIS calculations. Our identification of the levels fully agree with the one in the NIST [28] database and as well as with the one from semi-empirical data [6].

\section{References}

[1] Levchuk S, Maier H, Bolt H and Suzuki A 2007 J. Nucl. Mater. 3671033

[2] Carter W J 1966 Ph.D. Thesis (Johns Hopkins University)

[3] Krupke W F and Gruber J B 1963 J. Chem. Phys. 391024

[4] Krupke W F and Gruber J B 1964 J. Chem. Phys. 411225

[5] Vasile M, Avram N, Vlazan P, Grozescu I and Miclau M 2008 J. of Optoelectronics and Advanced Materials 102898

[6] Weber M J 1967 Phys. Rev. 157262

[7] Radžiūtė L, Gaigalas G, Kato D, Jönsson P, Rynkun P, Kučas S, Jonauskas V and Matulianec R, $J Q R S$ submitted 2014

[8] Johnson W R 2007 Atomic Structure Theory (New York: Springer)

[9] Jönsson P, Gaigalas G, Bieroń J, Froese Fischer C and Grant I P 2013 Comput. Phys. Commun. 1842197

[10] Grant I P 2007 Relativistic Quantum Theory of Atoms and Molecules (New York: Springer)

[11] McKenzie B J, Grant I P, and Norrington P H 1980 Comput. Phys. Commun. 21233

[12] Gaigalas G, Rudzikas Z, and Fischer Froese C 1997 J. Phys. B: At. Mol. Opt. Phys. 303747

[13] Gaigalas G, Žalandauskas T and Rudzikas Z 2003 At. Data Nucl. Data Tables 8499

[14] Gaigalas G, Žalandauskas T and Fritzsche S 2004 Comput. Phys. Commun. 157239

[15] Karazija R 1996 Introduction to the Theory of X-Ray and Electronic Spectra of Free Atoms (New York:Plenum Press)

[16] Karazija R and Kučas S 2013 JQSRT 129131

[17] Jonauskas V, Karazija R, and Kučas S 2008 J. Phys. B: At. Mol. Opt. Phys. 41215005

[18] Palaudoux J, Lablanquie P, Andric L, Ito K, Shigemasa E, Eland J H D, Jonauskas V, Kučas S, Karazija R and F. Penent F 2010 Phyd. Rev. A 82043419

[19] Jonauskas V, Kučas S and Karazija R 2011 Phyd. Rev. A 84053415

[20] Kučas S, Jonauskas V, Karazija R and Momkauskaite A 2007 Lithuan. J. Phys. 47249

[21] Jonauskas V, Kisielius R, Kynienė A, Kučas S and Norrington P H 2010 Phyd. Rev. A 81012506

[22] Jonauskas V, Gaigalas G, and Kučas S 2012 At. Data and Nucl. Data Tables 9819

[23] Cowan R D 1981 The Theory of Atomic Structure and Spectra (Berkeley, CA: University of California Press)

[24] Olsen J, Roos B O, Jorgensen P and Jensen H J Aa, 1988 J. Chem. Phys. 892185

[25] Sturesson L, Jönsson P and Froese Fischer C 2007 Comput. Phys. Commun. 177539

[26] Gaigalas G, Rudzikas Z and Fischer F C 1998 At. Data Nucl. Data Tables 701

[27] Radžiūtè L, Gaigalas G, Jönsson P and Bieroń J 2014 Phys. Rev. A 90012528

[28] Kramida A, Ralchenko Yu, Reader J, and NIST ASD Team 2013. NIST Atomic Spectra Database 
Energy level structure of the ground configuration in the $\mathrm{Er}^{3+}$ free ion

(ver. 5.1), [Online]. Available: http://physics.nist.gov/asd [2014, May 02]. National Institute of Standards and Technology, Gaithersburg, MD

180

[29] Fischer C F and G. Tachiev 2004 Atomic Data and Nucl. Data Tables 871

182 [30] Wybourne B G 1960 J. Chem. Phys. 32639 